\title{
Astrometric light-travel time signature of sources in nonlinear motion
}

\section{Derivation of the effect and radial motion}

\author{
G. Anglada-Escudé and J. Torra
}

Departament d'Astronomia i Meteorologia, Universitat de Barcelona, Av. Diagonal 647, 08028 Barcelona, Spain

e-mail: anglada@am.ub.es

Received 9 November 2005 / Accepted 6 December 2005

\section{ABSTRACT}

Context. Very precise planned space astrometric missions and recent improvements in imaging capabilities require a detailed review of the assumptions of classical astrometric modeling.

Aims. We show that Light-Travel Time must be taken into account in modeling the kinematics of astronomical objects in nonlinear motion, even at stellar distances.

Methods. A closed expression to include Light-Travel Time in the current astrometric models with nonlinear motion is provided. Using a perturbative approach the expression of the Light-Travel Time signature is derived. We propose a practical form of the astrometric modelling to be applied in astrometric data reduction of sources at stellar distances $(d>1 \mathrm{pc})$.

Results. We show that the Light-Travel Time signature is relevant at $\mu$ as accuracy (or even at mas) depending on the time span of the astrometric measurements. We explain how information on the radial motion of a source can be obtained. Some estimates are provided for known nearby binary systems

Conclusions. Given the obtained results, it is clear that this effect must be taken into account in interpreting precise astrometric measurements. The effect is particularly relevant in measurements performed by the planned astrometric space missions (GAIA, SIM, JASMINE, TPF/DARWIN). An objective criterion is provided to quickly evaluate whether the Light-Travel Time modeling is required for a given source or system.

Key words. astrometry - stars: kinematics - reference systems - time - binaries: general - planetary systems

\section{Introduction and notation}

Advances producing very precise astrometric measurements come essentially from space-borne astrometric missions like SIM (Shao 1998), GAIA (Perryman et al. 2001) or JASMINE (Gouda et al. 2002), and require a revision of the classic astrometric assumptions at many levels. Some of the concepts that are being reviewed carefully are those involving light signal propagation (Klioner 2003; Le Poncin-Lafitte et al. 2004) and the description of the astronomical sources and observers (Klioner 2004) in the context of the IAU resolutions (Soffel et al. 2003) that aim to define a consistent framework to model astronomical observations.

This effort involves many groups and individuals around the world and one relevant aspect is the appropriate description of stellar motion. This work focuses on the impact of LightTravel Time (LTT) on the observed direction of a source outside the solar system. Such considerations are as old as modern astronomy itself; in the 17th century Ole Römer used it to give the first estimation of the velocity of light.
In solar system dynamics, light travel delays are already widely considered and applied. Our aim is to show that it is also relevant for distant objects $(d>1 \mathrm{pc})$ and to develop analytic expressions to include the astrometric LTT signature in ultraprecise astrometric modeling. We will provide an expression which is algorithmically efficient and fully compatible with the IAU standards (Soffel et al. 2003) to the required level of accuracy. A full scheme of a more general astrometric model compatible with the BCRS is given in Klioner (2003). The present work may be seen as a refinement of the formulae given there to generalize stellar motion.

The first two sections are devoted to establishing the physical framework and defining the relevant quantities. Section 3 is devoted to determining the relation between the emission time interval $\Delta t_{\mathrm{e}}$ and the observation time interval $\Delta t_{\mathrm{obs}}$ to the required accuracy for astrometric purposes.

The baseline astrometric model is stated in Sect. 4.1 where the expression for a point source in linear motion is developed. There we define the concept of Linear Reference Motion (LRM) which will be very useful in the later developments. 
The same development for the source in linear motion was proposed by Klioner \& Kopeikin (1992), where the LTT due to the observer's position was already included. Light-Travel Time effects on sources in linear motion and their relation to constant radial velocities is a topic extensively discussed in the literature and directly related to apparent superluminal motion. A good review of this issue is found in Lindegren \& Dravins (2003). In Sect. 4.2, the astrometric model is naturally extended to sources in nonlinear motion. A more detailed study of radial nonlinear motion using spectroscopic measurements in the post-Newtonian framework can be found in Kopeikin \& Ozernoy (1999). Since the spectroscopic techniques are more sensitive to the local environment of the sources, a more sophisticated model using a larger set of reference systems is required there, and very precise information can be extracted.

Section 5 obtains a quantitative description of the astrometric LTT signature for a point source. The LTT signature appears as a second order correction as the astrometric radial velocity described in Lindegren \& Dravins (2003).

In Sect. 6, a series of examples show how the LTT signature carries information about the radial geometry of the trajectory of a source. This is of particular interest in binary systems or objects in Keplerian orbits(such as exoplanetary systems), because resolved LTT signatures may lead to the determination of the full set of orbital parameters without spectroscopic measurements, as is commonly required (see Batten 1973, Chap. 1).

Section 7 provides heuristic relations to evaluate the significance of the LTT effects in the geometrical characterization of any astronomical structure.

The use of LTT effects in epoch observations to determine properties of sources at stellar distances was first proposed by Irwin (1952), whose work is applicable to binary systems with at least one variable component. See Ribas et al. (2002) as an example of the use of this technique. Similar and more sophisticated models are used in precise pulsar timing. The timing measurements have been very fruitful in the field of millisecond pulsars in multiple systems. A pulsar works as an ultrastable clock and the time of arrival of the pulses can be measured very precisely in the radio range. A precise theoretical scheme using pulsar timming observations to test general relativity and obtain physical information of a given system is provided in Kopeikin (1995) and Kopeikin (1996). From the observational point of view, some of the most remarkable works are the detection of the first exoplanetary system around PSR1257+12 as reported by Wolszczan \& Frail (1992), and the tests of general relativity carried out observing PSR J0437-4715 by van Straten et al. (2001).

We summarize the most important notation and conventions used here.

- The velocity of light is $c$.

- The three-dimensional coordinate quantities ("3-vectors") referring to the spatial axes of the corresponding reference system are set in boldface italic: $\boldsymbol{a}=a^{i} \hat{\boldsymbol{e}}_{i}$, where $\left\{\hat{\boldsymbol{e}}_{i} ; i=\right.$ $1,2,3\}$ is the spatial basis defined by the coordinate system of the BCRS.
- The scalar product of two vectors $\boldsymbol{a}$ and $\boldsymbol{b}$ is denoted by $\boldsymbol{a} \cdot \boldsymbol{b}=\sum_{i=1}^{3} a^{i} b^{i}$.

- The Euclidean norm of a 3-vector $\boldsymbol{a}$ is denoted $\|\boldsymbol{a}\|$ and is computed as $\|\boldsymbol{a}\|=(\boldsymbol{a} \cdot \boldsymbol{a})^{1 / 2}$.

- $\langle\boldsymbol{a}\rangle$ means that the vector must be normalized using its own Euclidean norm $\langle\boldsymbol{a}\rangle=\frac{\boldsymbol{a}}{\|a\|}$.

- The components of the obtained vector using the cross product operation $\boldsymbol{a} \times \boldsymbol{b}$ are given by $(\boldsymbol{a} \times \boldsymbol{b})^{i}=\varepsilon_{i j k} a^{j} b^{k}$, where $\varepsilon_{i j k}$ is the completely antisymmetric Levi-Civita symbol with $\varepsilon_{x y z}=+1$.

- All physical quantities are expressed using the SI units (or MKS) if no particular comment is added in the text.

- Small angles are usually given in fractions of arcseconds. The most common shortcuts are mas $=10^{-3} "$ and $\mu$ as $=$ $10^{-6 \prime \prime}$.

- A symbol $p$ inside square brackets [...] after a symbol $f$, means that $f[p]$ is an explicit function of $p$. This notation is used thoughout the paper where the arguments in a function may appear ambiguous to the reader. Round brackets (...) are exclusively used to group algebraic expressions.

\section{Trajectories, quantities and reference system}

Our purpose is to determine the observed direction of a moving object from the position of an observer at rest with respect to the barycenter of the solar system in absence of gravitational fields. To do that, one must describe the motion of a source in terms of the observation instant $t_{\mathrm{obs}}$ instead of the emission instant $t_{\mathrm{e}}$. The relation between an emission time interval $\Delta t_{\mathrm{e}}$ and its corresponding observation time interval $\Delta t_{\mathrm{obs}}$ will be nonlinear and time dependent due to the nonlinear change of the distance a light signal must cover. This nonlinear time dependence will add additional apparent nonlinear terms to its motion on the celestial sphere.

We restrict the discussion to a particular inertial frame of special relativity where the space-time metric is assumed to be the Minkowsky metric with signature $(-+++)$. All the quantities and vectors refer to the BCRS spatial coordinates $x^{i}$ and the time coordinate $t$ describing the events is TCB - see Brumberg \& Groten (2001). The BCRS metric is not a Minkowsky metric, but since the astrometric LTT signature is already very small, the gravitational light bending and the kinematical aberration can be treated as a posteriori effects to the observed direction (see Klioner 2003). The trajectory of a point source is described as a Linear Reference Motion $\boldsymbol{x}_{\mathrm{LRM}}[t]$ plus a nonlinear shift $\boldsymbol{D}[t]$. The spatial part of the trajectory of the source in BCRS coordinates is given by

$$
\begin{aligned}
\boldsymbol{x}_{\mathrm{s}}[t] & =\boldsymbol{x}_{\mathrm{LRM}}[t]+\boldsymbol{D}[t], \\
\boldsymbol{x}_{\mathrm{LRM}}[t] & =\boldsymbol{x}_{\mathrm{LRM}}^{0}+\boldsymbol{v}_{\mathrm{LRM}}^{0}\left(t-t_{\mathrm{e}}^{0}\right),
\end{aligned}
$$

where $\boldsymbol{x}_{\mathrm{LRM}}^{0}$ are the coordinates of the LRM at some instant $t_{\mathrm{e}}^{0}$. This initial instant will be discussed more precisely below. Formally, the constant velocity term $\boldsymbol{v}_{\mathrm{LRM}}^{0}$ could be included in $\boldsymbol{D}[t]$, but it is very useful to keep it apart in order to define properly the Barycentric astrometric parameters (see Sect. 4.1) and relate them to the physical quantities in (1). As an example, $\boldsymbol{x}_{\text {LRM }}^{0}$ and $\boldsymbol{v}_{\text {LRM }}^{0}$ describe the motion of the center of mass of a 


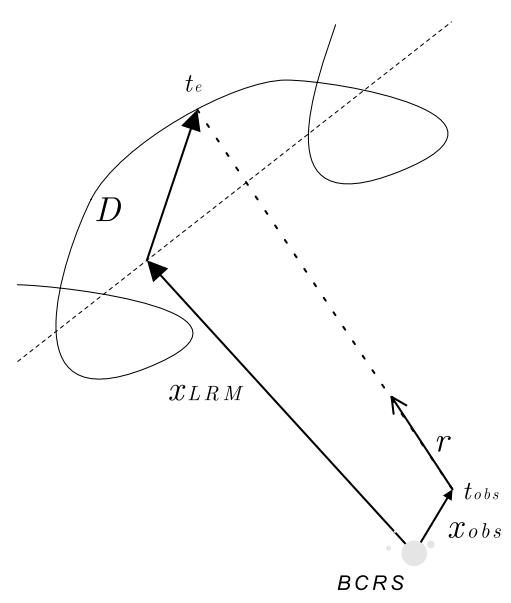

Fig. 1. Scheme of the vectors involved in the computation of the observed direction at a given event of observation. The vector $\boldsymbol{x}_{\mathrm{LRM}}$ is used to define a fiducial Linear Reference Motion, which could be, for example, the trajectory of the center of mass of a binary system. The shift vector $\boldsymbol{D}$ is the nonlinear contribution to the motion. Using again the example of the binary systems, it might be the orbital motion of a component around the center of mass of the system. The little vector $\boldsymbol{r}$ is the observed direction of the incoming light ray at the event of observation.

binary system and $\boldsymbol{D}$ describes the orbital motion of one of the components.

The value of coordinate time $t$ at the emission event $E$ is denoted by $t_{\mathrm{e}}=t[E]$. The spatial coordinates $\boldsymbol{x}_{\mathrm{s}}$ at the emission event (which coincide with the spatial coordinates of the source) are denoted by $\boldsymbol{x}_{\mathrm{s}}\left[t_{\mathrm{e}}\right]$. In the same way the value of the coordinate time at the observation event is $t$ [Obs] $=t_{\mathrm{obs}}$ and the spatial coordinates of such an event are $\boldsymbol{x}_{\mathrm{obs}}\left[t_{\mathrm{obs}}\right]$. Please note that $t_{\mathrm{e}}$ and $t_{\mathrm{obs}}$ are both given in the same time scale, which is TCB.

Since in Minkowsky space-time the light rays follow straight lines, the spatial vector joining an event of emission at $t_{\mathrm{e}}$ and an event of observation at $t_{\mathrm{obs}}$ defines the observed unit direction as

$\boldsymbol{r}=\left\langle\boldsymbol{x}_{\mathrm{s}}\left[t_{\mathrm{e}}\right]-\boldsymbol{x}_{\mathrm{obs}}\left[t_{\mathrm{obs}}\right]\right\rangle$.

For the later developments, we need to identify the small time intervals

$O(1) \sim \frac{\boldsymbol{v}_{\mathrm{LRM}}^{0} \Delta t}{c} ; \frac{\boldsymbol{D}}{c} ; \frac{\boldsymbol{x}_{\mathrm{obs}}}{c}$.

The vectors in (4) have dimensions of time and have typical absolute values going from some minutes to several hours or days (or even years). From now on, the module $\left\|x_{\text {LRM }}^{0}\right\|$ will be written as $x_{\text {LRM }}$ to simplify the notation. It is also useful to introduce the small adimensional quantities

$O(1) \sim \frac{v_{\mathrm{LRM}}^{0} \Delta t}{x_{\mathrm{LRM}}} ; \frac{\boldsymbol{D}}{x_{\mathrm{LRM}}} ; \frac{\boldsymbol{x}_{\mathrm{obs}}}{x_{\mathrm{LRM}}}$

Relations (5) impose that the initial distance between the source and the observer $x_{\text {LRM }}$ must be much larger than all the other time-dependent displacements $\boldsymbol{v}_{\mathrm{LRM}}^{0} \Delta t, \boldsymbol{D}$ and $\boldsymbol{x}_{\mathrm{obs}}$. This requirement is usual in the astrometric modelling of objects beyond the solar system. We define the barycentric reference direction $\boldsymbol{l}_{\mathbf{0}}$ as the unit vector

$\boldsymbol{l}_{\mathbf{0}}=\frac{\boldsymbol{x}_{\mathrm{LRM}}^{0}}{x_{\mathrm{LRM}}}$,

which is the direction towards the position of the LRM (i.e. the center of mass of a binary system) given by an observer at the BCRS origin at a given reference instant $t_{\mathrm{obs}}^{0}$, usually called barycentric reference epoch.

With the exception of the initial direction parameterized through two angles, only the parameters producing time dependent changes in the observed direction will produce measurable effects. Despite the frequent appearance of $t_{\mathrm{e}}^{0}$ throughout the paper, this parameter is not directly measurable. The physical quantities producing time dependent effects are $x_{\mathrm{LRM}}, \boldsymbol{v}_{\mathrm{LRM}}^{0}$, $D$. They depend only on $t_{\mathrm{e}}^{0}$ in their formal definition implicitly given in (1).

\section{Equation of time delay}

In the most general case, the interval of time between the event of observation and the event of emission is related to the spatial coordinate distance as

$$
\begin{aligned}
\left(t_{\mathrm{obs}}-t_{\mathrm{e}}\right)= & \frac{1}{c}\left\|\boldsymbol{x}_{\mathrm{obs}}\left[t_{\mathrm{obs}}\right]-\boldsymbol{x}_{\mathrm{s}}\left[t_{\mathrm{e}}\right]\right\| \\
& +\Delta_{\mathrm{BCRS}}\left[t_{\mathrm{e}}, t_{\mathrm{obs}}\right]+\Delta_{\mathrm{ext}}\left[t_{\mathrm{e}}, t_{\mathrm{obs}}\right] \\
& +O(2) .
\end{aligned}
$$

The $\Delta$ terms on the right side can include additional space-time effects due to gravitational contributions due to the BCRS fields $\left(\Delta_{\mathrm{BCRS}}\right)$ and other external fields $\Delta_{\text {ext }}$ that the photon may feel along its long trajectory. This gravitational contribution is usually known as the Shapiro effect. For a detailed discussion of the Shapiro effect see Kopeikin \& Schäfer (1999). We are interested in the relation between the emission interval $\Delta t_{\mathrm{e}}$ and the observation interval $\Delta t_{\mathrm{obs}}$. Then, using the relation (7) for two different events of emission $E^{0}$ and $E$ and their respective observation events $\mathrm{Obs}^{0}$ and Obs we obtain

$$
\begin{aligned}
\Delta t_{\mathrm{obs}}-\Delta t_{\mathrm{e}}= & \frac{\left\|\boldsymbol{x}_{\mathrm{obs}}\left[t_{\mathrm{obs}}\right]-\boldsymbol{x}_{\mathrm{s}}\left[t_{\mathrm{e}}\right]\right\|}{c} \\
& -\frac{\left\|\boldsymbol{x}_{\mathrm{obs}}\left[t_{\mathrm{obs}}^{0}\right]-\boldsymbol{x}_{\mathrm{s}}\left[t_{\mathrm{e}}^{0}\right]\right\|}{c} \\
& +\Delta_{\mathrm{BCRS}}\left[t_{\mathrm{e}}, t_{\mathrm{obs}}\right]-\Delta_{\mathrm{BCRS}}\left[t_{\mathrm{e}}^{0}, t_{\mathrm{obs}}^{0}\right] \\
& +\Delta_{\mathrm{ext}}\left[t_{\mathrm{e}}, t_{\mathrm{obs}}\right]-\Delta_{\mathrm{ext}}\left[t_{\mathrm{e}}^{0}, t_{\mathrm{obs}}^{0}\right],
\end{aligned}
$$

$$
\begin{aligned}
\Delta t_{\mathrm{e}} & =t_{\mathrm{e}}-t_{\mathrm{e}}^{0}, \\
\Delta t_{\mathrm{obs}} & =t_{\mathrm{obs}}-t_{\mathrm{obs}}^{0} .
\end{aligned}
$$

For most of the stars the absolute value of $\Delta_{\text {ext }}$ may be very large since the gravitational fields of the galaxies and other mass distributions may contribute; however, in most circumstances, it will not change significantly during the lifetime of a 
space astrometric mission (even over some hundreds of years). An exception may be objects orbiting large concentrations of mass or gravitational lensing events. In such cases, the model for the observations must be carefully derived not only from the point of view of the LTT. This might be the case for stars moving close to the Milky way's central black hole (see Ghez et al. 1998). An example of such a detailed model is found in Fragile \& Mathews (2000). Despite the use of such models to include very sophisticated space-time effects, the astrometric LTT signature due to the nonlinear motion is ignored. The contributions $\Delta_{\mathrm{BCRS}}$ will heavily depend on the relative position of the observer and the sources of gravitational fields (i.e. Sun, planets). Considering that the physical diameter of such bodies is some orders of magnitude larger that their Schwarschild radius, this Shapiro term adds a very small time shift (a few milliseconds at most). In such an interval of time, the direction of observation will not change more than a few nanoarcseconds which is, by far, an undetectable astrometric quantity with current techniques. From now on we will omit both $\Delta$ terms.

The equation of time delay (8) depends on the module of the relative position of the observer and the source, and in general, it cannot be used to obtain a closed exact expression of $\Delta t_{\mathrm{e}}$ in terms of $\Delta t_{\mathrm{obs}}$. This is due the nonlinear nature of the module operations on the right side of Eq. (8) and the intrinsically nonlinear dependence of $\boldsymbol{x}_{\mathrm{s}}$ with respect to time. A perturbative approach is chosen here to obtain the lowest order contribution that is relevant enough to produce an astrometric shift at $\mu a s$ level of accuracy. With some algebra whose details are given in Appendix A, and using the definitions of small quantities provided in Eqs. (4), (5) it is obtained that, at first order

$\Delta t_{\mathrm{e}}=$

$\alpha_{\mathrm{s}}\left(\Delta t_{\mathrm{obs}} \cdot-\frac{1}{c} \boldsymbol{l}_{\mathbf{0}} \cdot \Delta \boldsymbol{D}\left[t_{\mathrm{obs}}\right]+\frac{1}{c} \boldsymbol{l}_{\mathbf{0}} \cdot \Delta \boldsymbol{x}_{\mathrm{obs}}\left[t_{\mathrm{obs}}\right]\right)+\boldsymbol{O}(2)$.

In Eq. (11) some notation shortcuts are applied. These are

$$
\begin{aligned}
\alpha_{\mathrm{s}} & =\frac{1}{1+\boldsymbol{l}_{\mathbf{0}} \cdot \frac{\boldsymbol{v}_{\mathrm{LRM}}^{0}}{c}}, \\
\boldsymbol{\Delta} \boldsymbol{x}_{\mathrm{obs}}\left[t_{\mathrm{obs}}\right] & =\boldsymbol{x}_{\mathrm{obs}}\left[t_{\mathrm{obs}}\right]-\boldsymbol{x}_{\mathrm{obs}}\left[t_{\mathrm{obs}}^{0}\right], \\
\Delta \boldsymbol{D}\left[t_{\mathrm{obs}}\right] & =\boldsymbol{D}\left[t_{\mathrm{e}}^{0}+\alpha_{\mathrm{s}} \Delta t_{\mathrm{obs}}\right]-\boldsymbol{D}\left[t_{\mathrm{e}}^{0}\right] .
\end{aligned}
$$

The factor $\alpha_{\mathrm{s}}$ multiplying the full expression (11) is the one responsible for apparent superluminal velocities.

\section{Astrometric model for point-like sources}

The aim of this section is to provide expressions that complete the astrometric models at $\mu$ as accuracy incorporating the LTT. Under the assumptions of Sects. 2 and 3, the observed direction of a point-like source given by an observer at rest at the event of observation is

$$
\boldsymbol{r}\left[t_{\mathrm{obs}}, t_{\mathrm{e}}\right]=\left\langle\boldsymbol{x}_{\mathrm{LRM}}^{0}+\boldsymbol{v}_{\mathrm{LRM}}^{0} \Delta t_{\mathrm{e}}+\boldsymbol{D}\left[t_{\mathrm{e}}^{0}+\Delta t_{\mathrm{e}}\right]-\boldsymbol{x}_{\mathrm{obs}}\left[t_{\mathrm{obs}}\right]\right\rangle
$$

The next subsections provide general purpose parametric expressions to be used in the astrometric model for a point-like source outside of the solar system. We applied the formalism of the local triad as described in Murray (1983). Section 4.2 extends the astrometric model to sources in nonlinear motion including the LTT amplitudes. At this point, the expression of the observed direction (15) depends on the emission instant $t_{\mathrm{e}}$.We will include the LTT in the astrometric model using just the relation between $\Delta t_{\mathrm{e}}$ and $\Delta t_{\mathrm{obs}}$ given by Eqs. (11) in (15).

\subsection{Linear reference motion}

This is the simple case where the source is moving in linear motion (i.e. a single star). Substituting (11) in (15) and imposing $\boldsymbol{D}[t]=0$ for any instant of time $t$, we obtain

$$
\begin{aligned}
& \boldsymbol{r}_{\mathrm{LRM}}\left[t_{\mathrm{obs}}\right]=\left\langle\boldsymbol{l}_{\mathbf{0}}\left(1+\mu_{r 0} \Delta T_{\mathrm{LRM}}\right)+\left(\mu_{\delta 0} \boldsymbol{p}+\mu_{\alpha 0}^{*} \boldsymbol{q}\right) \Delta T_{\mathrm{LRM}}\right. \\
& \left.+\boldsymbol{\pi}\left[t_{\mathrm{obs}}\right]\right\rangle \\
& \boldsymbol{p}=\left(-\sin \delta_{0} \cos \alpha_{0},-\sin \delta_{0} \sin \alpha_{0}, \cos \delta_{0}\right), \\
& \boldsymbol{q}=\left(-\sin \alpha_{0}, \cos \alpha_{0}, 0\right), \\
& \boldsymbol{l}_{\mathbf{0}}=\left(\cos \alpha_{0} \cos \delta_{0}, \sin \alpha_{0} \cos \delta_{0}, \sin \delta_{0}\right) \\
& \mu_{\alpha 0}^{*}=\mu_{\alpha 0} \cos \delta_{0}=\frac{\alpha_{\mathrm{s}} \boldsymbol{v}_{\mathrm{LRM}}^{0} \cdot \boldsymbol{q}}{x_{\mathrm{LRM}}} \\
& \mu_{\delta 0}=\frac{\alpha_{\mathrm{s}} \boldsymbol{v}_{\mathrm{LRM}}^{0} \cdot \boldsymbol{p}}{x_{\mathrm{LRM}}}, \\
& \mu_{r 0}=\frac{\alpha_{\mathrm{s}} \boldsymbol{v}_{\mathrm{LRM}}^{0} \cdot \boldsymbol{l}_{\mathbf{0}}}{x_{\mathrm{LRM}}}, \\
& \boldsymbol{\pi}\left[t_{\mathrm{obs}}\right]=\frac{\boldsymbol{x}_{\mathrm{obs}}\left[t_{\mathrm{obs}}\right]}{x_{\mathrm{LRM}}}=\frac{\prod_{0}}{A U} \boldsymbol{x}_{\mathrm{obs}}\left[t_{\mathrm{obs}}\right] \\
& \Delta T_{\mathrm{LRM}}=\Delta t_{\mathrm{obs}}+\frac{1}{c} \boldsymbol{l}_{\mathbf{0}} \cdot \Delta \boldsymbol{x}_{\mathrm{obs}}\left[t_{\mathrm{obs}}\right]
\end{aligned}
$$

These definitions extend those given for the HIPPARCOS catalog (see Perryman et al. 1997, Vol. 1) and include the Roemer correction due to observer's motion, which was already introduced by Klioner \& Kopeikin (1992). The vectors $\boldsymbol{p}$ and $\boldsymbol{q}$ are unit vectors tangent to the celestial sphere at $\boldsymbol{l}_{\mathbf{0}}$ direction, pointing towards the direction of increasing declination and right ascension respectively. The quantities $\alpha_{0}, \delta_{0}, \Pi_{0}, \mu_{\alpha 0}^{*}, \mu_{\delta 0}$ and $\mu_{r 0}$, are the so-called barycentric astrometric parameters for a point-like source in rectilinear motion at the barycentric reference epoch $t_{\mathrm{obs}}^{0}$. The angles $\alpha_{0}$ and $\delta_{0}$ are the right ascension and the declination of the equatorial coordinate system given in radians. The parameter $\Pi_{0}$ is the parallax in radians. The symbol $\mu_{\alpha 0}^{*}$ is the proper motion in the $\alpha_{0}$ direction multiplied by $\cos \delta_{0}$, which provides the correct angular shift taking into account the distortion of the spherical coordinates towards the poles, and $\mu_{\delta 0}$ is the proper motion in the declination direction; both expressed in $\mathrm{rad} \mathrm{s}^{-1}$. The parameter $\mu_{r 0}$ is known as astrometric radial velocity (see Lindegren \& Dravins 2003) given in $\mathrm{s}^{-1}$. AU is the Astronomical Unit which is currently defined as a constant.

\subsection{Nonlinear motion}

The expression that generalizes to point-like sources in nonlinear motion is straightforward. The six astrometric 
parameters described in Sect. 4.1 are used to define a fiducial LRM while $\boldsymbol{D}$ contains the nonlinear contributions,

$$
\begin{aligned}
\boldsymbol{r}\left[t_{\mathrm{obs}}\right]= & \left\langle\boldsymbol{l}_{\mathbf{0}}\left(1+\mu_{r} \Delta T\right)\right. \\
& +\left(\mu_{\delta 0} \boldsymbol{p}+\mu_{\alpha 0}^{*} \boldsymbol{q}\right) \Delta T \\
& \left.+\frac{\boldsymbol{D}\left[t_{\mathrm{e}}^{0}+\alpha_{\mathrm{s}} \Delta T\right]}{x_{\mathrm{LRM}}}+\boldsymbol{\pi}\left[t_{\mathrm{obs}}\right]\right\rangle,
\end{aligned}
$$

$$
\Delta T=\Delta t_{\mathrm{obs}}-\frac{1}{c} \boldsymbol{l}_{\mathbf{0}} \cdot\left(\Delta \boldsymbol{D}\left[t_{\mathrm{obs}}\right]-\Delta \boldsymbol{x}_{\mathrm{obs}}\left[t_{\mathrm{obs}}\right]\right) .
$$

These expressions are sufficient to include LTT in an astrometric data reduction algorithm at $\mu$ as. In classic astrometry, the LTT terms with $\boldsymbol{D}$ inside $\Delta T$ were safely neglected since the astrometric measurements were not precise enough. In the next section we will analyze the effect of this LTT term on the observed direction, which is the LTT astrometric signature.

\section{Analytic estimation of the LTT signature}

To estimate the astrometric LTT signature we need to compare Eq. (25) with the classical approach for the observed direction $\boldsymbol{r}_{\mathrm{c}}$. As the classical approach we define

$$
\begin{aligned}
\boldsymbol{r}_{\mathrm{c}}= & \left\langle\boldsymbol{l}_{\mathbf{0}}\left(1+\mu_{r} \Delta T_{\mathrm{c}}\right)+\left(\mu_{\delta 0} \boldsymbol{p}+\mu_{\alpha 0}^{*} \boldsymbol{q}\right) \Delta T_{\mathrm{c}}\right. \\
& \left.+\frac{\boldsymbol{D}\left[t_{\mathrm{e}}^{0}+\alpha_{\mathrm{s}} \Delta T_{\mathrm{c}}\right]}{x_{\mathrm{LRM}}}+\boldsymbol{\pi}\left[t_{\mathrm{obs}}\right]\right\rangle, \\
\Delta T_{\mathrm{c}}= & \Delta t_{\mathrm{obs}} ;
\end{aligned}
$$

where the difference with respect to Eq. (25) is essentially in $\Delta T_{\mathrm{c}}$, which classically does not include the LTT contribution due to the nonlinear motion of the source with respect to the LRM, and due to the position of the observer with respect to the barycenter of the solar system. This last contribution (Römer term) is already contained in recent accurate astrometric models (Klioner \& Kopeikin 1992), but we prefer to also consider it here for completeness. The comparison of $\boldsymbol{r}$ and $\boldsymbol{r}_{\mathrm{c}}$ is performed by direct subtraction of (25) and (27) up to $O(2)$, considering $O(1)$ all those terms containing expressions proportional to those in Eqs. (4), (5). Then, the astrometric LTT signature $\delta \boldsymbol{r}$ is defined as

$\delta \boldsymbol{r}=\boldsymbol{r}\left[t_{\mathrm{obs}}\right]-\boldsymbol{r}_{\mathrm{c}}\left[t_{\mathrm{obs}}\right]$,

and, after some algebra

$$
\delta \boldsymbol{r}=\boldsymbol{l}_{\mathbf{0}} \times\left(\boldsymbol{\delta} \times \boldsymbol{l}_{\mathbf{0}}\right)+O(3)
$$

$\boldsymbol{\delta}=-\alpha_{\mathrm{S}} \frac{\boldsymbol{v}_{\mathrm{LRM}}^{0}+\boldsymbol{V}_{\mathrm{orb}}\left[t_{\mathrm{e}}^{0}+\alpha_{\mathrm{s}} \Delta t_{\mathrm{obs}}\right]}{x_{\mathrm{LRM}}} \Delta T_{\mathrm{LTT}}$

$\Delta T_{\mathrm{LTT}}=\frac{\boldsymbol{l}_{\mathbf{0}} \cdot\left(\Delta \boldsymbol{D}\left[t_{\mathrm{obs}}\right]-\Delta \boldsymbol{x}_{\mathrm{obs}}\left[t_{\mathrm{obs}}\right]\right)}{c}$.

The presence of the orbital velocity vector $\boldsymbol{V}_{\text {orb }}$ is justified in Appendix A. It is called orbital velocity by direct analogy with the binary system. The LTT shift $\delta \boldsymbol{r}$ is an astrometric detectable quantity since it is in the perpendicular direction to the line of sight $\boldsymbol{l}_{\mathbf{0}}$, as is explicit in Eq. (30). The LTT shift depends on the radial projection of the orbital motion $\boldsymbol{l}_{\mathbf{0}} \cdot \Delta \boldsymbol{D}$. This implies that using accurate astrometric measurements one would, in principle, be able to constrain the radial motion of the source or, on the other hand, if some information of the radial motion of the source is provided (i.e. radial velocities), the fit of the astrometric orbit might be more robust and accurate. In Sect. 6, the relevancy of the astrometric LTT signature will be shown in some well known multiple systems. The apparent position of a source is advanced or delayed with respect to the nonretarded trajectory. That is why the expression (31) is just the instantaneous proper motion multiplied by the time interval $\Delta T_{\mathrm{LTT}}$, which is also time dependent.

In formula (31) two velocities appear instead of the instantaneous total velocity. They are kept separate since the ways they affect the observed direction (their astrometric signature) have quite different properties. The proper motion term $\boldsymbol{v}_{\mathrm{LRM}}^{0}$ is constant and the time dependence of this part of the astrometric LTT signature will come only from the variations of the $\Delta T_{\text {LTT }}$ interval. However the orbital velocity $\boldsymbol{V}_{\text {orb }}$ is intrinsically time dependent, periodic in most cases, and its coupling with the $\Delta T_{\mathrm{LTT}}$ interval will produce a more sophisticated astrometric signature. As shown in Sect. 6, when applied to binary systems, the scaling law of each contribution with respect to the orbital elements is significantly different.

In addition, the term $\boldsymbol{D}\left[t_{\mathrm{e}}^{0}\right]$ may be included in $\boldsymbol{x}_{\mathrm{LRM}}^{0}$ by imposing $\boldsymbol{D}\left[t_{\mathrm{e}}^{0}\right]=0$. However, this assumption may not be useful in practical cases (i.e. when applied to fitting the orbital parameters of a binary system). We will keep the current expression unless we need to implement LTT in the particular modelling of an object.

The LTT signature is one among other astrometric effects becoming relevant at second order astrometric accuracy. These effects include perspective acceleration (or astrometric radial velocity), and all the couplings of the parallax with the proper motion or the non-linear motion. A comprehensive list can be found in Dravins et al. (1999). All of them are naturally included in (16) and (25), since our expressions are directly derived from the kinematical model of the source. Expanding Eq. (25) up to $O(2)$ in the small terms (5), the vectorial expressions of all such contributions are explicitly obtained. Any astrometric study aiming to obtain information using any second order contribution must properly consider the LTT signature explained in this work.

\section{Some numerical estimates}

Let us naively use the expression (31) to obtain some order of magnitude estimates of the LTT signature. The semiamplitudes (denoted by $\overline{\delta \boldsymbol{r}}$ ) of the astrometric LTT signatures for a component in a binary system in circular orbit can be estimated as

$$
\begin{aligned}
& \overline{\boldsymbol{\delta}}_{\text {proper }} \sim 15.812 \mu \text { as } \frac{a^{\prime \prime} \mu_{\text {mas } / \text { year }}}{\pi_{\text {mas }}} \sin i, \\
& \overline{\boldsymbol{\delta}}_{\text {orbital }} \sim 99353 \mu \text { as } \frac{a^{\prime \prime 2}}{\pi_{\text {mas }} P_{\text {year }}} \sin i .
\end{aligned}
$$




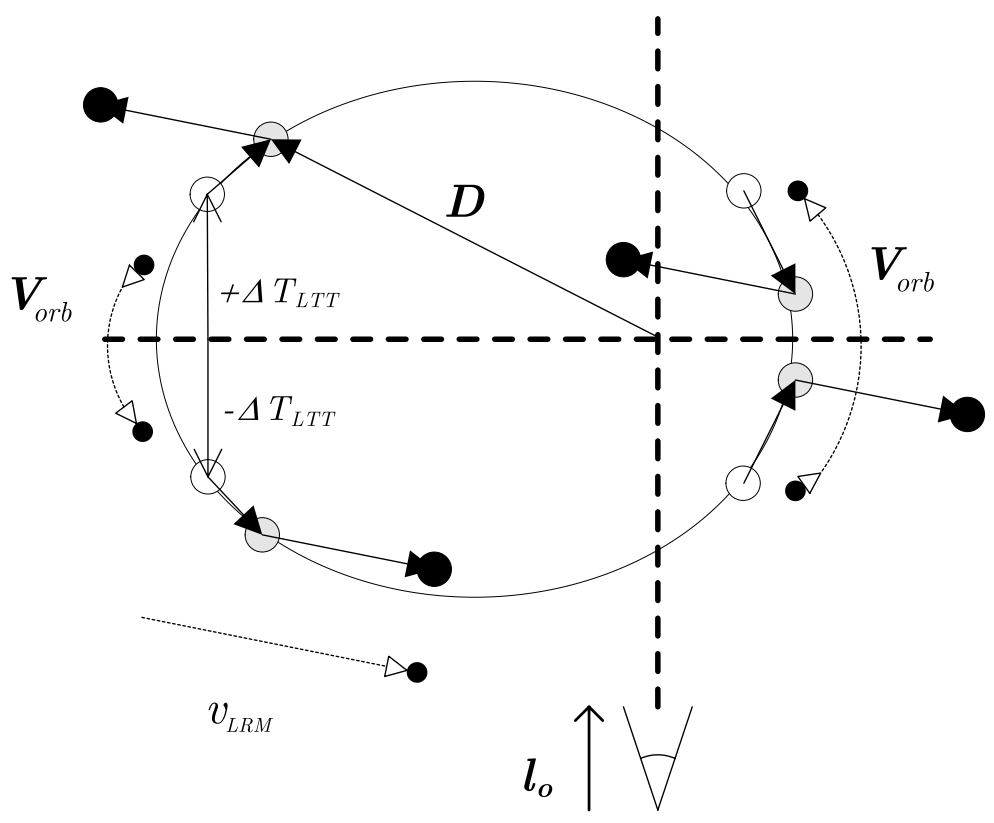

Fig. 2. Astrometric LTT signature in a binary system. The elliptic orbital motion of a companion is shown in the case where the inclination is $90 \mathrm{deg}$. The horizontal dashed line is the semi-major axis of the orbit and is perpendicular to the line of sight given by $\boldsymbol{l}_{\mathbf{0}}$. The white circles represent the nonretarded positions of the star at different moments of the orbit. The gray circles are the apparent positions corrected by the Light-Travel Time appliying only the LTT correction due to the instantaneous orbital velocity $\boldsymbol{V}_{\text {orb }}$. The black circles are the final apparent position after also considering the motion of the barycenter of the binary system. The proper motion of the system is omitted to simplify the visualization of both the LTT signatures. The pure orbital correction $V_{\text {orb }} \Delta T_{\text {LTT }}$ changes its orientation continuously depending on the orbital position(gray circles) and it is larger when the object is closer to the center of mass of the system (right side of the figure). On the other hand, the proper motion contribution $\boldsymbol{v}_{\mathrm{LRM}}^{0} \Delta T_{\mathrm{LTT}}$ (dark circles) always contributes in the same direction changing only the sign and the amplitude of the perturbation. The projection of the shifts on the plane perpendicular to the line of sight are the astrometrically measurable quantities.

For simplicity, these expressions are obtained imposing $\Delta \boldsymbol{x}_{\mathrm{obs}}=0$. Relations (33), (34) are provided using catalog-like parameters where $a^{\prime \prime}$ is the projected semi-major axis in arcseconds, $\mu_{\text {mas/year }}$ is the proper motion module in mas year ${ }^{-1}$ and $\pi_{\text {mas }}$ is the parallax in mas. The effect is modulated by the inclination of the orbit.

Kepler's third law can be used to obtain the expressions (33) and (34) as powers of the orbital period $P$ and the orbital semimajor axis $R$. It is found that $\overline{\boldsymbol{\delta}}_{\text {proper }}$ scales as $R^{1}$ (or $P^{2 / 3}$ ), while $\overline{\boldsymbol{\delta}}_{\text {orbital }}$ scales as $R^{1 / 2}$ (or $P^{1 / 3}$ ). This illustrates that for systems with long period orbits the LTT signature due to the coupling with the proper motion will be more significant. This is expected since $\boldsymbol{v}_{\mathrm{LRM}}^{0}$ does not depend on the semimajor axis of the orbit(it is only related to the velocity of the center of mass of the system), while $\boldsymbol{V}_{\text {orb }}$ becomes smaller at larger orbital distances (i.e. in the solar system, distant planets have lower orbital velocities).

The semiamplitudes obtained using (33) and (34) for some nearby systems are provided in Table 1.

A detailed model to include the astrometric LTT signature in a strict orbital solution of binary systems will be provided in a later work, since some detailed discussion of the definition of the orbital parameters must be provided to keep the astrometric model accuracy at the $\mu$ as level.

As shown in Table 1, long period binaries usually have larger LTT signatures coming from the coupling with the proper motion term. This is the case of $61 \mathrm{Cyg}$. In that situation, the LTT signature could be resolved using available long-term lower precision astrometry.

\section{Conclusions}

We have shown that the LTT must be taken into account to obtain accurate models of the observable quantities in precise modern astrometry. Furthermore, since LTT is an objectdependent effect, precise relative astrometric measurements are sufficient to resolve the astrometric LTT signatures. The astrometric LTT signature may be used in highly precise groundbased observations to obtain additional information on a given object. The expressions derived in (30)-(31) can be applied using $\mathrm{O}-\mathrm{C}$ techniques with the available astrometric data. LTT must be taken into account in the interpretation of data obtained by the planned space astrometric missions which that will attempt to reach astrometric accuracies of a few $\mu$ as. As an example, the coupling of the Römer delay with the proper motion and the orbital velocity of a star can mimic the astrometric wobble caused by a planetary mass object with a period of around one year (considering an observer traveling on the vicinity of the earth) if LTT is not properly included in the astrometric model.

It has been found that the LTT signature is boosted by the proper motion of the system. Multiple systems with high proper motions (thick disk, globular clusters, nearby halo objects) might be objects of investigation if properly adapted astrometric models are used. 
Table 1. The data in this table were prepared using the WDS Sixth Catalog of Orbits of Visual Binary Stars (Hartkopf et al. 2004) and the HIPPARCOS catalog (Perryman et al. 1997). The value of some of the orbital parameters shown are average values. The numbers shown here are orientative since the circular model applied is very unrealistic. It can be seen that both contributions, $\delta \boldsymbol{r}_{\text {proper }}$ and $\delta \boldsymbol{r}_{\text {orbital }}$, may have quite different signification depending on the binary system. In very eccentric systems, like $\alpha$ Cen, the orbital term can be considerably larger than the numbers shown here around the perihelion. Another feature that is blurred by the applied approximations is that if the components of the system have different masses, each component might show very different astrometric LTT signatures. The values for AB pic-b are very approximated and are based on very recent and sparse data of this candidate to planetary system (Chauvin et al. 2005). This values are added here as an example of the applicability of the astrometric LTT signature to general purpose orbital solutions. In this case, the LTT signature would not be very useful to improve the orbital solution due to the very long period and tininess of the signal.

\begin{tabular}{lccccccc}
\hline \hline System & $\begin{array}{c}\text { Period } \\
\text { years }\end{array}$ & $\begin{array}{c}a^{\prime \prime} \\
\text { arcsec }\end{array}$ & $\begin{array}{c}i \\
\text { deg }\end{array}$ & $\begin{array}{c}\text { Parallax } \\
\text { mas }\end{array}$ & $\begin{array}{c}\text { Proper motion } \\
\text { mas/year }\end{array}$ & $\begin{array}{c}\Delta \sigma_{\text {proper }} \\
\mu \text { as }\end{array}$ & $\begin{array}{c}\Delta \sigma_{\text {orbital }} \\
\mu \text { as }\end{array}$ \\
\hline 61 Cyg & 722.0 & 14.9 & 51.85 & 294 & 5227 & 3293 & 81.7 \\
$\alpha$ Cen & 79.9 & 8.75 & 79 & 742 & 3672 & 670 & 415 \\
HD 110314 & 3.09 & 0.021 & 122.9 & 14 & 191.9 & 3.80 & 0.86 \\
HD 2475 & 5.65 & 0.146 & 64 & 118 & 31.01 & 0.54 & 2.85 \\
AB pic-b & $\sim 3000$ & 0.753 & $? ?$ & 21.97 & 47.36 & $\sim 50$ & $\sim 1$ \\
\hline
\end{tabular}

If the astrometric LTT signature is large, information on the radial geometry of a system can be obtained. In the case of binary systems this information allows us to solve or at least constrain, the full set of orbital elements without information about radial velocities. Despite that, the amplitudes appearing in Table 1 are rather small, even at $\mu a s$ accuracy level. It seems unfeasible to use the astrometric LTT signatures to significantly improve the knowledge of a given system since radial velocity curves are much better measured. The suggestion here is to use the radial velocity measurements as an input to improve the astrometry of a given source using the LTT-corrected orbital description. How to proceed properly in such cases is under investigation.

When imaging capabilities of exoplanetary systems become available, the fitting of an LTT orbit might lead to constraining the size of the orbit in the line-of-sight direction without the use of radial velocity measurements, which may be impracticable on such faint objects unless interferometric techniques improve significantly.

The relations (33)-(34) can be used as a good indicator of whether LTT astrometric effects must be taken into account for more general objects (open clusters, globular clusters, galaxies, fast orbiters around massive black holes) in nonlinear motion. For this purpose we define the LTT astrometric signal as

$\mathcal{L}_{\mathrm{TT}} \equiv \frac{V}{c} \frac{L}{d}$

where $V$ and $L$ are the characteristic velocity and longitude of the system, respectively; $c$ is the speed of light in the same units as $V$; and $d$ is an estimation of the distance to the source given in the same units as $L . \mathcal{L}_{\mathrm{TT}}$ is an adimensional quantity that can be directly interpreted as an angle(in radians). If this number is of the order of the astrometric accuracy used to describe an astronomical object outside the solar system, then the LTT should be taken into account in order to give a correct interpretation of the astrometric data.

For some purposes (such as the construction of an astrometric catalog) it is sometimes useful to make the abstraction that at the reference epoch $t_{\mathrm{obs}}^{0}$ the observer is located at the barycenter of the Solar System. If this is done, the initial direction $\boldsymbol{r}\left[t_{\mathrm{obs}}^{0}\right]$ will not depend on the Römer delay $\boldsymbol{l}_{\mathbf{0}} \cdot \boldsymbol{x}_{\mathrm{obs}}\left[t_{\mathrm{obs}}^{0}\right]$ due to the observer position at $t_{\mathrm{obs}}^{0}$ as it does in expressions (24) and (26) or in the astrometric LTT signature formula (31). Then we can substitute $\boldsymbol{x}_{\mathrm{obs}}\left[t_{\mathrm{obs}}\right]-\boldsymbol{x}_{\mathrm{obs}}\left[t_{\mathrm{obs}}^{0}\right]$ by simply $\boldsymbol{x}_{\mathrm{obs}}\left[t_{\mathrm{obs}}\right]$ in both equations (24) and (26) or in (31). This consideration is very useful to create an astrometric catalog for a given reference epoch independently of the relative initial position of observer $\boldsymbol{x}_{\mathrm{obs}}\left[t_{\mathrm{obs}}^{0}\right]$ and the barycentric reference direction of each source $\boldsymbol{l}_{\mathbf{0}}$.

A precise modelling of the LTT to be applied in the astrometry of binary systems is being developed and will be presented in a later publication.

Acknowledgements. This work was carried with the financial support of grant ESP2003-04352 of the Ministry of Education and Science, Spain. The authors thank I.Ribas and S.A.Klioner their comments. We thank the anonymous referee the helpful report. All the members of the GAIA group in Barcelona are gratefully acknowledged.

\section{Appendix A: First order derivation of equation of time delay}

The first term on the right side of the equation (8), to first order as defined in (4)-(5), reads

$$
\begin{gathered}
\frac{\left|\boldsymbol{x}_{\mathrm{obs}}\left[t_{\mathrm{obs}}\right]-\boldsymbol{x}_{\boldsymbol{e}}\left[t_{\mathrm{e}}\right]\right|}{c} \simeq \frac{1}{c} x_{\mathrm{LRM}}+\boldsymbol{l}_{\mathbf{0}} \cdot \frac{\boldsymbol{v}_{\mathrm{LRM}}^{0} \Delta t_{\mathrm{e}}}{c}+\boldsymbol{l}_{\mathbf{0}} \cdot \frac{\boldsymbol{D}\left[t_{\mathrm{e}}\right]}{c} \\
-\boldsymbol{l}_{\mathbf{0}} \cdot \frac{\boldsymbol{x}_{\mathrm{obs}}\left[t_{\mathrm{obs}}\right]}{c}+O(2) .
\end{gathered}
$$

The second term of the right hand of (8) is straightforward using $t_{\mathrm{e}}^{0}$ instead of $t_{\mathrm{e}}$ in the last expression (A.1). After some algebra the emision time interval $\Delta t_{\mathrm{e}}$ can be written as

$$
\begin{aligned}
& \Delta t_{\mathrm{e}}=\frac{1}{1+\boldsymbol{l}_{\mathbf{0}} \cdot \frac{\boldsymbol{v}_{\mathrm{LRM}}^{0}}{c}} \\
& \times\left(\Delta t_{\mathrm{obs}}-\boldsymbol{l}_{\mathbf{0}} \cdot \frac{\boldsymbol{D}\left[t_{\mathrm{e}}\right]-\boldsymbol{D}\left[t_{\mathrm{e}}^{0}\right]}{c}+\boldsymbol{l}_{\mathbf{0}} \cdot \frac{\boldsymbol{x}_{\mathrm{obs}}\left[t_{\mathrm{obs}}\right]-\boldsymbol{x}_{\mathrm{obs}}\left[t_{\mathrm{obs}}^{0}\right]}{c}\right) \\
& +O(2) \text {. }
\end{aligned}
$$


The term multiplying the full expression is responsible for apparent superluminal velocities. For this reason we call this term superluminal factor

$\alpha_{\mathrm{S}}=\frac{1}{1+\boldsymbol{l}_{\mathbf{0}} \cdot \frac{\boldsymbol{v}_{\mathrm{LRM}}^{0}}{c}}$.

In spite of the suppression of the second order terms there is still a dependency on $t_{\mathrm{e}}$ on the right hand of Eq. (A.2) in $\boldsymbol{D}$. This equation is enough to solve $\Delta t_{\mathrm{e}}$ iteratively. But our purpose is to obtain a closed form accurate to $O(1)$. To solve this we consider

$$
\begin{aligned}
\boldsymbol{D}\left[t_{\mathrm{e}}\right]-\boldsymbol{D}\left[t_{\mathrm{e}}^{0}\right]=\boldsymbol{D}\left[t_{\mathrm{e}}^{0}+\alpha_{\mathrm{s}} \Delta t_{\mathrm{obs}}+\delta t\right]-\boldsymbol{D}\left[t_{\mathrm{e}}^{0}\right], \\
\delta t=-\alpha_{\mathrm{S}} \boldsymbol{l}_{\mathbf{0}} \cdot \frac{\boldsymbol{D}\left[t_{\mathrm{e}}\right]-\boldsymbol{D}\left[t_{\mathrm{e}}^{0}\right]}{c} \\
\quad+\alpha_{\mathrm{S}} \boldsymbol{l}_{\mathbf{0}} \cdot \frac{\boldsymbol{x}_{\mathrm{obs}}\left[t_{\mathrm{obs}}\right]-\boldsymbol{x}_{\mathrm{obs}}^{0}\left[t_{\mathrm{obs}}^{0}\right]}{c} \sim O(1) .
\end{aligned}
$$

Taking this into account, we can write to first order in $\delta t$

$$
\begin{aligned}
& \frac{\boldsymbol{D}\left[t_{\mathrm{e}}\right]-\boldsymbol{D}\left[t_{\mathrm{e}}^{0}\right]}{c}=\frac{\boldsymbol{D}\left[t_{\mathrm{e}}^{0}+\alpha_{\mathrm{s}} \Delta t_{\mathrm{obs}}\right]-\boldsymbol{D}\left[t_{\mathrm{e}}^{0}\right]}{c} \\
& +\frac{\boldsymbol{V}_{\mathrm{orb}}\left[t_{\mathrm{e}}^{0}+\alpha_{\mathrm{s}} \Delta t_{\mathrm{obs}}\right]}{c} \delta t+O(2) .
\end{aligned}
$$

In Eq. (A.6) the term $\frac{V_{\text {orb }}}{c} \delta t$ is of $O(2)$ and will be neglected in (A.2). The development (A.6) is also used to justify the appearance of $\boldsymbol{V}_{\text {orb }}$ in equation (31). These are all the ingredients needed to obtain the relation of $\Delta t_{\mathrm{e}}$ in terms of $\Delta t_{\mathrm{obs}}$ to first order

$$
\begin{aligned}
\Delta t_{\mathrm{e}}= & \alpha_{\mathrm{s}}\left(\Delta t_{\mathrm{obs}}-\boldsymbol{l}_{\mathbf{0}} \cdot \frac{\boldsymbol{D}\left[t_{\mathrm{e}}^{0}+\alpha_{\mathrm{s}} \Delta t_{\mathrm{obs}}\right]-\boldsymbol{D}\left[t_{\mathrm{e}}^{0}\right]}{c}\right. \\
& \left.+\boldsymbol{l}_{\mathbf{0}} \cdot \frac{\left.\boldsymbol{x}_{\mathrm{obs}}\left[t_{\mathrm{obs}}\right]\right)-\boldsymbol{x}_{\mathrm{obs}}\left[t_{\mathrm{obs}}^{0}\right]}{c}\right)+\boldsymbol{O}(2)
\end{aligned}
$$

which is the same as Eq. (11) with the suitable notation shortcuts explained in Eqs. (12)-(14).

\section{References}

Batten, A. H. 1973, Binary and Multiple Systems of Stars, Vol. textbook (Chicago: Pergamon Press Ltd.)

Brumberg, V. A., \& Groten, E. 2001, A\&A, 367, 1070

Chauvin, G., Lagrange, A.-M., Zuckerman, B., et al. 2005, A\&A, 438, L29

Dravins, D., Lindegren, L., \& Madsen, S. 1999, A\&A, 348, 1040

Fragile, P. C., \& Mathews, G. J. 2000, ApJ, 542, 328

Fridlund, C. V. M. 2000, ESA Bulletin, 103, 20

Ghez, A. M., Klein, B. L., Morris, M., \& Becklin, E. E. 1998, ApJ, 509,678

Gouda, N., Tsujimoto, T., Kobayashi, Y., et al. 2002, Ap\&SS, 280, 89

Hartkopf, W. I., Mason, B. D., \& Worley, C. E. 2004, US Naval Observatory web page, 12 July 2004 Release

Irwin, J. B. 1952, ApJ, 116, 211

Klioner, S. A. 2003, AJ, 125, 1580

Klioner, S. A. 2004, Phys. Rev. D, 69, 124001

Klioner, S. A., \& Kopeikin, S. M. 1992, AJ, 104, 897

Kopeikin, S. M. 1995, ApJ, 439, L5

Kopeikin, S. M. 1996, ApJ, 467, L93

Kopeikin, S. M., \& Ozernoy, L. M. 1999, ApJ, 523, 771

Kopeikin, S. M., \& Schäfer, G. 1999, Phys. Rev. D, 60, 124002

Le Poncin-Lafitte, C., Linet, B., \& Teyssandier, P. 2004, Classical and Quantum Gravity, 21, 4463

Lindegren, L., \& Dravins, D. 2003, A\&A, 401, 1185

Makarov, V. V., \& Kaplan, G. H. 2005, AJ, 129, 2420

Murray, S. S. 1983, Extragalactic Astronomy, Science Year (Chicago: World Book)

Perryman, M. A. C., de Boer, K. S., Gilmore, G., et al. 2001, A\&A, 369, 339

Perryman, M. A. C., Lindegren, L., Kovalevsky, J., et al. 1997, A\&A, 323, L49

Ribas, I., Arenou, F., \& Guinan, E. F. 2002, AJ, 123, 2033

Shao, M. 1998, in Astronomical Interferometry, ed. R. D. Reasenberg, Proc. SPIE, 3350, 536

Soffel, M., Klioner, S. A., Petit, G., et al. 2003, AJ, 126, 2687

van Straten, W., Bailes, M., Britton, M., et al. 2001, Nature, 412, 158

Wolszczan, A., \& Frail, D. A. 1992, Nature, 355, 145 